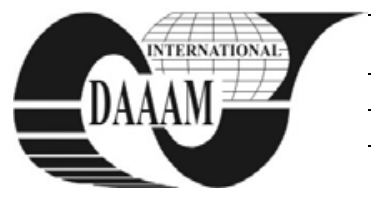

Annals of DAAAM for 2011 \& Proceedings of the 22nd International DAAAM Symposium, Volume 22, No. 1, ISSN 1726-9679 ISBN 978-3-901509-83-4, Editor B. Katalinic, Published by DAAAM International, Vienna, Austria, EU, 2011 Make Harmony between Technology and Nature, and Your Mind will Fly Free as a Bird Annals \& Proceedings of DAAAM International 2011

\title{
RESEARCH OF MEMS SENSOR BASED ON MECHANICAL FILTER BANK
}

\author{
DUSEK, D[aniel]
}

\begin{abstract}
This paper deal with research of a MEMS sensor for decomposition of mechganical signals based on a mechanical filter bank. Comparison with today used method of mechanical signals decomposition and basic mathematical model of the mechanical filter bank is described first.Then also main areas of using (vibro diagnostics, speech processing and cochlear implants) are described
\end{abstract}

Key words: MEMS, sensor, vibrations, acoustics

\section{INTRODUCTION}

Goal of this work is research of a new sensor for sensing and decomposition of mechanical sinals based on mechanical filter bank. This work is inspired by function of human or generally mammalian ear concretelly by function of a cochlea. The cochlea is that part of inner ear where mechanical signals (acoustic pressure) into electrical signals are converted and consequently decomposed into simple frequency components.

The decomposition of the signals into simple frequency components is made just thanks to mechanical filtration. This effect is evoked thanks to special modal properties of basilar membrane where auditory sense cells are located. If the basilar membrane is actuated by low frequencies maximum amplitude of vibration of basilar membrane is located on its apical end and if the basilar membrane is actuated by high frequencies maximum amplitude of vibration of basilar membrane is located on its basal end. It means that every section of the basilar membrane correspond to different frequency. This effect was observed firs by von Békésy already in the fifties of $20^{\text {th }}$ century (Békésy, 1960).

The decomposition of mechanical signals like acoustic pressure or vibrations by the mechanical filter bank can bring any advantages compared to classical methods of the mechanical signals decompositions.

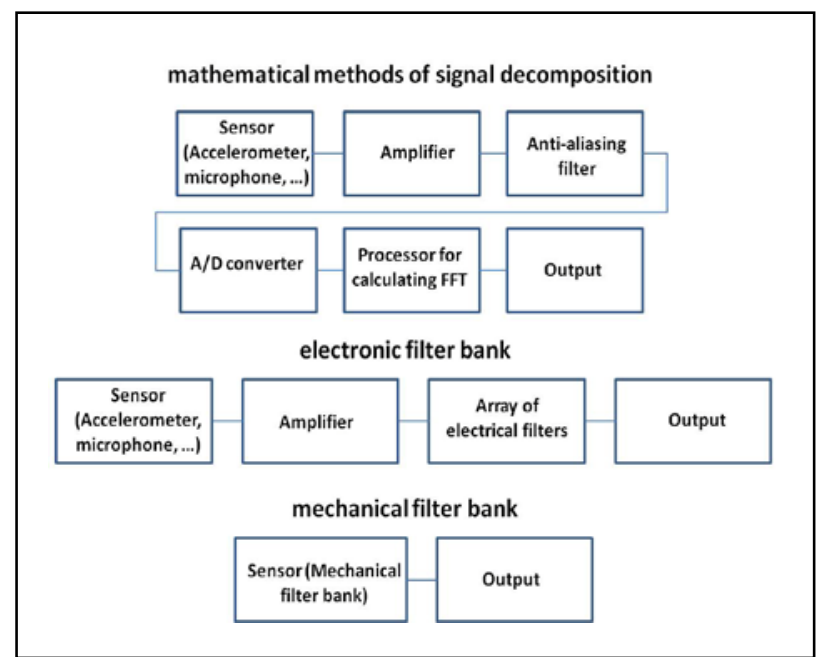

Fig. 1. Comparison of measurement chains for different methods of mechanical signals decomposition
Today two basic principles of decomposition of the mechanical signals are used. First principle is based on mathematical methods like Fast Fourier Transformation (FFT) or wavelet transformation.

Second principle of mechanical signal decomposition is based on electronic filter banks. Basic measurement chains of the today used methods of mechanical signals decomposition compared with mechanical filter bank are shown in the figure 1 .

Using of the mechanical filter bank markedly reduces the measurement chain which brings following advantages:

- Reduction of electronic circuits should evokes lower additional noise

- $\quad$ Reduction of electrical circuits should leads to lower power supply

- $\quad$ The MEMS mechanical filter bank would have been more sensitive because the signals are amplified by mechanical resonance (classical sensors like microphones or accelerometers working in principle bellow resonance and the signal must be amplified in amplifier)

Today technologies like Micro Electro Mechanical Systems (MEMS) or Nano Electro Mechanical Systems (NEMS) which could produce the mechanical filter bank in very small dimensions are very fast growing. Today already mainly technologies MEMS are ordinarily used in many technical applications like MEMS accelerometers, MEMS gyroscopes and many more (Gad-el-Hak, 2006).

Big advantage of the MEMS devices compared to classical sensors is their markedly lower price and also possibility of their integration together with external electrical circuits on one chip.

The goal of this work is just research and development of the mechanical filter bank for using in the said areas of using which could be produced by any of the MEMS technologies.

\section{MATHEMATICAL MODEL}

A basic scheme of signal decomposition by the mechanical filter bank is shown in the figure 2. A principle of the signal decomposition is very simple. The mechanical filter bank is basically an array of mechanical resonators with different eigenfrequencies.

If the array of resonators is actuated by any mechanical signals so only that resonators start resonate whose eigenfrequency is equal to the frequency component composed in the signal.

The array of resonators (filters) can be described by following differential equation:

$$
\mathrm{M} \mathrm{q} \mathrm{q}^{*}+\mathrm{B} \mathrm{q}^{\cdot}+\mathrm{Kq}=\mathrm{q}_{\mathrm{z}} \cdot \mathrm{B}+\mathrm{q}_{\mathrm{z}} \mathrm{K}+\mathrm{K}_{\mathrm{s} 1} \mathrm{q}+\mathrm{K}_{\mathrm{s} 2} \mathrm{q}
$$

Where $\mathbf{q}_{\mathbf{z}}$ is a vector of displacement of excitation, $\mathbf{q}_{\mathbf{z}}{ }^{\cdot}$ is a vector of velocity of excitation, $\mathbf{q}$ is a vector of displacement of resonators, $\mathbf{q}$ is a vector of velocity of resonators and $\mathbf{q}^{*}$ is a 


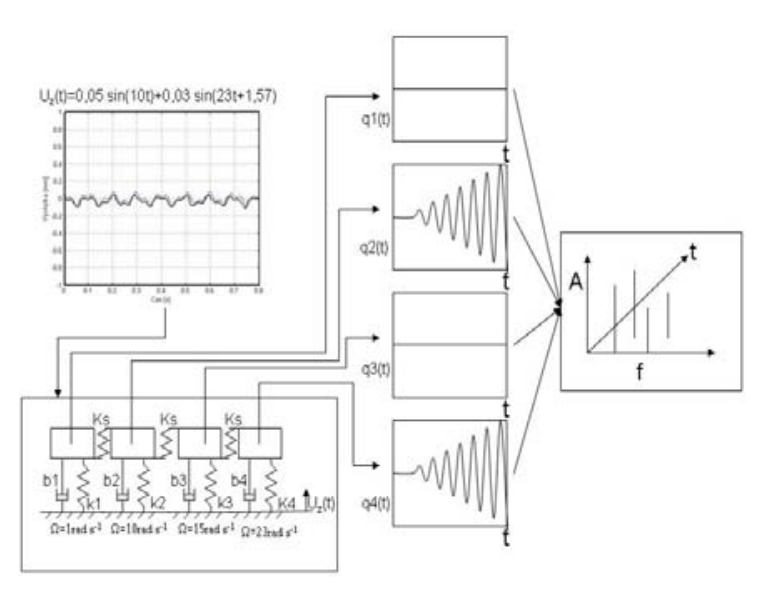

Fig. 2. Basic scheme of signal decomposition by mechanical filter bank

vector of acceleration of resonators. $\mathbf{M}$ is a matrix of mass of the resonators, $\mathbf{B}$ is a matrix of damping of the resonators, $\mathbf{K}$ is a matrix of stiffness of resonators and $\mathbf{K}_{\mathrm{s} 1}$ and $\mathbf{K}_{\mathrm{s} 2}$ are matrixes of stiffness of connection sprint between neighbour resonators.

Main results calculated on the mathematical model are not shown here but they were published in previous years in papers (Dusek, 2009) or (Dusek, 2010).

\section{APPLICATIONS OF THE NEW SENSOR}

The MEMS sensor based on the mechanical filter bank could be used in many technical applications where decomposition of mechanical signals is useful. There are three main areas of possible usage:

\section{On-line machine diagnostics based on vibration monitoring:}

The machine dignostics based on vibrafon monitoring is very effective instrument for monitoring of the machine condition and for prediction and prevention of possible wrecking states. The on-line diagnostics makes possible to observe the state of the machine continually in real time.

Today the diagnostics and especially on-line diagnostics based on vibrafon monitoring is very expensive and for this reason is used mainly for monitoring of large turbo sets in power plants or similar areas.

The MEMS mechanical filter bank should be able to expand also to other technical areas thanks to its low price (for example into automotive industry, aircraft industry, machine tools and etc.). There are also good possibilities for using of any energy harvesting method for supply of the sensor and makes it fully independent on external energy sources.

\section{Speech processing and voice control of technical objects:}

The higher level speech processing is based on analising of frequency spectrums of the signal. Today mathematical methods like Fast Fourier or Wavelet transformations (or together with cepstral analysis) are used. But it needs complicated measurement chain (figure 1) and very high requirements on processor which calculate the spectrums. The MEMS mechanical filter bank is on the contrary very simple and also the price of the MEMS device is great advantage compared to today using methods of speech processing.

Today the speech processing and voice kontrol of technical object is highly increasing area. The voice kontrol can help disabled people and also makes control of technical objects much more comfortable. Thanks to its low price, low power requirements and simplicity the MEMS mechanical filter bank could be used also in many applications where it is today impossible (for example voice control of household appliances, control of any car systems and etc.).
Cochlear implants:

The Cochlear implants are special devices which replace function of damaged or functionless human cochlea. Today the cochlear implants are composed from two basic parts- external and internal. The external part is placed out of head and includes microphone, electronic circuits for signal decomposition (speech processsor) and transmitter. The internal part is implanted into the head and includes receiver with support electronic circuits and electrodes which are connected to hearing nerves (Rubinstein, 2004), (Wikipedia, 2010). Today also first experiments with tottaly implantable implants are realized but results are not still good as the classical cochlear implants. Also any scientists on the world try to design the artificial cochlea like MEMS micro mechanical sensor (Shintaku et al., 2010).

Main advantages of the MEMS mechanical filter bank compared to today used systems is low power requirements which together with using of energy harvesting systems could lead to totally implantable implants without needing of any external energy source (The totally implantable implants described previously need implantable battery).

\section{CONCLUSION}

This paper deals with possibilities of using of MEMS mechanical filter bank for decomposition of mechanical signals like vibrations or acoustic pressure. The MEMS mechanical filter bank sensor offers some advantages opposite to today used method of the mechanical signals decomposition. Low price of MEMS sensor is main advantage. Simplicisity of the sensor and possibilities of full integration on one chip together with electronical circuit is also great advantage of the sensor. A low power requirement of the new MEMS sensor is also very important feature which could lead to design of fully independent sensor on external energy sources.

\section{ACKNOWLEDGEMENTS}

The paper was written with the support by the Grant Agency of AS CR - Project No.: KJB201730802 and by the EU project No.: CZ.1.07/2.2.00/07.0406.

\section{REFERENCES}

Békésy, G von. (1960). Experiments in Hearing, McGraw-Hill, ISBN 978-0070043244, New York

Dusek, D. (2009). A Mathematical Model of an Artificial Cochlea Based on an Array of Resonators, In: DAAAM International Scientific Book, Katalinic, B. (Ed.), pp. 273280, DAAAM International, ISBN 978-3-901509-69-8, Vienna, Austria

Dusek, D. (2010). Design of a MEMS artificial cochlea like an array of resonators, Proceedings of 21th International $D A A A M$ Symposium, conference data, Viena, ISSN, ISBN 978-3-901509-73- 5, Katalinic, B. (Ed.), , DAAAM International, Vienna

Gad-el-Hak, M. (2006). The MEMS Handbook, CRC Press, ISBN 0-8493-9138-5, Boca Raton

Rubinstein, J. T. (2004). How cochlear implants encode speech, Current Opinion in Otolaryngology \& Head and Neck Surgery, Vol. 12, No. 5, pp. 444-448, ISSN 1068-9508

Shintaku, H.; Nakagava T.; Kitigawa, D.; Tanuyaja, H.; Kawano, S.; Ito, J. (2010) Development of piezoelectric acoustic sensor with frequency selectivity for artificial cochlea, Sensors and Actuators A: Physical, Vol. 158, Issue 2, March 2010, pp. 183-192, ISSN 0924-4247

*** (2010) http://en.wikipedia.org/wiki/Cochlear_implantWikipedia The Free Encyclopaedia, Accessed on:2010-5-25 